\title{
Action of a force near the planar surface between two semi-infinite immiscible liquids at
} very low Reynolds numbers

\author{
K. Aderogba and J.R. Blake
}

\begin{abstract}
Explicit expressions for the Green's functions due to a point force in one of two half space fluids are presented for the case when inertial effects of the fluid are negligible (Stokes flow) and the interface between the two fluids is considered to be flat due to the action of surface tension. The analytic expressions are discussed in terms of singularity diagrams. For the case of a force parallel to the interface a first approximation to the interface displacement is made.
\end{abstract}

\section{Introduction}

Many problems arise where we need to consider the movement of a particle, or particles, near the material surface between two immiscible liquids. Clearly, this problem is important in studies of surface phenomena in colloid and suspension mechanics. In this paper we look at a highly idealised part of this general and difficult problem. We study the flow-fields in both half spaces due to the action of a point force in one of them. The equations of motion used in our analysis are for very low Reynolds number (Stokes flow) and we assume that the material surface between the two liquids is almost flat due to the action of surface tension. For the case of the force acting parallel to the interface we can

Received 21 January 1978. 
obtain a first order approximation to the interface shape.

We suppose there is a distinct, sharp boundary between the two fluids even though in reality there will be a "mixed" region a few molecules thick where there will be continuous variation in the physical properties. In this paper we assume the interface is not surface active, so the properties of the surface can be characterised by a constant surface tension. Thus we have a benign, well-behaved, sharp interface between the liquids, a situation which we clearly do not attain in many problems in colloid science or membrane physiology.

A vast amount of literature has been published in recent years on colloid science, suspension mechanics, and membrane physiology, but very much less material on the more esoteric topic of this paper. In the next section, a review of some of the recent literature on the subject of this paper is presented. Later sections concentrate on analytic expressions for the Green's function, a description of the resulting expression in terms of singularity diagroms, and a first order approximation to interface shape for the case of a force parallel to the zeroth-order approximation to the interface (that is the plane $x_{3}=0$ ).

\section{Review of earlier work}

Most studies of flow fields around particles at very low Reynolds numbers have been concerned with infinite fluids, that is infinite in all directions. Semi-infinite or half-space fluids have attracted a more restricted attention (for an excellent review of older material, see Happel, Brenner [5]). Recently there have been several studies on the motion of slender bodies in a semi-infinite fluid (de Mestre [9], Blake [2], de Mestre, Russell [10], Lighthill [8], Katz, Blake and PaveriFontana [6]). All of these have been based on the Green's function for a point force in Stokes flow near a plane no-slip boundary which was stated explicitly by Blake [2], and reproduced here for a fluid in the negative half space $\left(x_{3}<0\right)$, 
(1a) $u_{i}=\frac{F_{j}}{8 \pi \mu}\left[\frac{\delta_{i j}}{r}+\frac{r_{i}{ }^{r}}{r^{3}}-\left(\frac{\delta_{i j}}{R}+\frac{R_{i}{ }^{R} j}{R^{3}}\right)\right.$

$$
\left.+2 h\left(\delta_{j \alpha} \delta_{\alpha k}{ }^{-\delta}{ }_{j 3} \delta_{3 k}\right) \frac{\partial}{\partial R_{k}}\left\{\frac{h R_{i}}{R^{3}}+\frac{\delta_{i 3}}{R}+\frac{R_{i} R}{R^{3}}\right\}\right] \text {, }
$$

(Ib) $\quad p=\frac{F}{4 \pi}\left[\begin{array}{l}r_{i} \\ -\frac{i}{3}\end{array}-\frac{R_{j}}{R^{3}}+2 h\left(\delta_{j \alpha} \delta_{\alpha k}-\delta_{j 3} \delta_{3 k}\right) \frac{\partial}{\partial R_{k}}\left[\frac{R_{3}}{R^{3}}\right)\right]$,

where

$$
r=\left[x_{1}^{2}+x_{2}^{2}+\left(x_{3}+h\right)^{2}\right]^{\frac{1}{2}}
$$

and

$$
R=\left[x_{1}^{2}+x_{2}^{2}+\left(x_{3}-h\right)^{2}\right]^{\frac{2}{2}}
$$

where $i, j$, and $k$ can take values of 1,2 , or $3, \alpha$ is restricted to 1 or 2 and the summation convention is assumed. We use the notation: $u$ representing the velocity vector, $p$ the pressure, and $F$ the point force located at $(0,0,-h)$. The no-slip boundary condition is at $x_{3}=0$, so that $R$ is the distance from the image point $(0,0, h)$.

So far this review has centred on no-slip boundaries and this is principally because so little analysis has been concerned with the interface between two fluids. Blake [3] stated the fundamental singularity plus image system (that is Green's function) for a point force in a semiinfinite fluid $\left(x_{3}<0\right)$ bounded by a planar free surface (at $x_{3}=0$ ) as

$$
\begin{aligned}
& u_{i}=\frac{F}{8 \pi \mu}\left[\frac{\delta_{i j}}{r}+\frac{r_{i}{ }^{r} j}{r^{3}}+\left(\delta_{j \alpha} \delta_{\alpha k}-\delta_{j 3} \delta_{3 k}\right)\left(\frac{\delta_{i k}}{R}+\frac{R_{i} R}{R^{3}}\right)\right], \\
& p=\frac{F}{4 \pi}\left[\frac{{ }^{r}}{r^{3}}+\left(\delta_{j \alpha} \delta_{\alpha k}-\delta_{j 3} \delta_{3 k}\right) \frac{R_{k}}{R^{3}}\right] .
\end{aligned}
$$

Here we are supposing that surface tension is sufficiently large that the free surface is approximately flat.

Recently Aderogba [1] has obtained the solution for the velocity and pressure in two adjoining half-space fluids in terms of a set of differential operators on the infinite fluid solution. He used the method 
of Papkovitch-Neuber potentials to obtain the differential operator form of the solution. However, in his model he used the matching conditions that the velocities be continuous across the interface and not the additional condition that the normal velocity also be equated to zero. This is the case if we have surface tension acting on the interface, and will be the case in the theory developed in this paper.

\section{Equations of motion and boundary conditions}

To model the flow-fields in Regions I and II due to the action of a point force in fluid I (see Figure 1) we will consider the following equations.

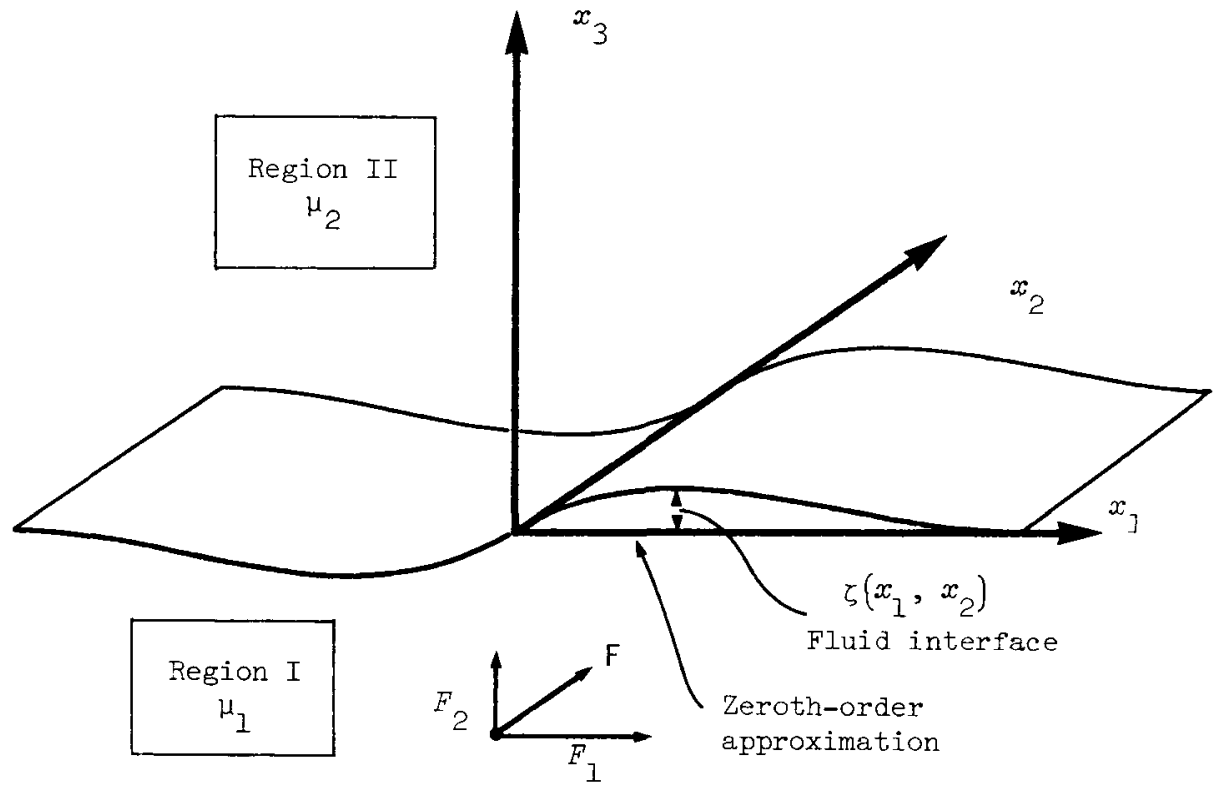

FIGURE 1. Illustrates the two half spaces used in analysis and some of the notation used in the text.

In Region I

$$
\begin{aligned}
& \nabla p^{(1)}=\mu_{1} \nabla^{2} u^{(1)}+F \delta(x-h), \\
& \nabla . u^{(I)}=0,
\end{aligned}
$$


where $h=(0,0,-h)$ and where the subscript or superscript (1) refers to Region I.

In Region II

$$
\begin{aligned}
& \nabla p^{(2)}=\mu_{2} \nabla^{2} u^{(2)}, \\
& \nabla . u^{(2)}=0 .
\end{aligned}
$$

In (3) and (4), $p$ refers to pressure, $u$ the velocity vector, $F \delta(x-h)$ the point force, and $\mu$ the viscosity. We assume that the displacement of the interface $x_{3}=\zeta\left(x_{1}, x_{2}\right)$ is small in comparison to $h$, the only other length scale in the problem. Thus we can obtain the zeroth order solution by equating the boundary conditions on the planar surface $x_{3}=0$. The conditions are:

$$
u_{\alpha}^{(1)}=u_{\alpha}^{(2)} ; \alpha=1,2 \text {, }
$$

$$
u_{3}^{(1)}=u_{3}^{(2)}=0
$$

$$
\mu_{1}\left(\frac{\partial u_{\alpha}^{(1)}}{\partial x_{3}}+\frac{\partial u_{3}^{(1)}}{\partial x_{\alpha}}\right)=\mu_{2}\left(\frac{\partial u_{\alpha}^{(2)}}{\partial x_{3}}+\frac{\partial u_{3}^{(2)}}{\partial x_{\alpha}}\right) ; \alpha=1,2 .
$$

Equations ( $5 a$ ) and $(5 b)$ are the kinematic conditions, with ( $5 b$ ) coming from the condition that $x_{3}=\zeta\left(x_{1}, x_{2}\right)$ be a material surface and the subsequent small amplitude approximation to obtain the zeroth order solution. Equation ( $5 \mathrm{c}$ ) is the dynamic condition that the tangential stresses are continuous. The first order approximation for the interface displacement $\zeta$ comes from the balance of normal stresses at the interface, that is the jump in normal stress must be balanced by surface tension. Thus, for small displacements, the equation becomes (see Landau and Lifshitz [7]),

$$
T \nabla_{2}^{2} \zeta=\left[p^{(1)}-p^{(2)}+2\left[\mu_{2} \frac{\partial u_{3}^{(2)}}{\partial x_{3}}-\mu_{1} \frac{\partial u_{3}^{(1)}}{\partial x_{3}}\right]\right]_{x_{3}=0},
$$

where $T$ is the surface tension and $\nabla_{2}^{2}$ is the two dimensional laplacian 
in $x_{1}$ and $x_{2}$.

The analysis is straightforward and may be obtained in several ways, either by Fourier transform methods (see Blake [2]), or by using Papkovitch-Neuber methods (see Aderogba [1]).

\section{Solutions and interpretation}

Using the notation of $(1 \mathrm{a}),(1 \mathrm{~b}),(2 \mathrm{a}),(2 \mathrm{~b})$, the analytic expressions for the velocity and pressure are as follows:

In Region I $\left(x_{3}<0\right.$, includes forcing stokeslet)

$$
\begin{aligned}
u_{i}^{(1)}=\frac{E_{j}}{8 \pi \mu_{1}}\left[\frac{\delta_{i j}}{r}+\right. & \frac{r_{i}{ }^{p} j}{r^{3}}+\left(\frac{1-\theta}{1+\theta} \delta_{j \alpha} \delta_{\alpha k}-\delta_{j 3} \delta_{3 k}\right)\left(\frac{\delta_{i k}}{R}+\frac{R_{i} R_{k}}{R^{3}}\right) \\
& \left.+\frac{2 \theta}{1+\theta} h\left(\delta_{j \alpha} \delta_{\alpha k}{ }^{-\delta_{j 3} \delta_{3 k}}\right) \frac{\partial}{\partial R_{k}}\left\{\frac{h R_{i}}{R^{3}}+\frac{\delta_{i 3}}{R}+\frac{R_{i} R_{3}}{R^{3}}\right\}\right],
\end{aligned}
$$

(Tb) $p^{(1)}=\frac{F_{j}}{4 \pi}\left[\frac{r_{j}}{\underline{r}^{3}}+\left(\frac{1-\theta}{1+\theta} \delta_{j \alpha} \delta_{\alpha k}-\delta_{j 3} \delta_{3 k}\right) \frac{R_{j}}{R^{3}}\right.$

$$
\left.+\frac{2 \theta}{1+\theta} h\left(\delta_{j \alpha} \delta_{\alpha k}^{-\delta_{j 3} \delta_{3 k}}\right) \frac{\partial}{\partial R_{k}}\left(\frac{R_{3}}{R^{3}}\right)\right] \text {. }
$$

In Region II $\left(x_{3}>0\right)$

$$
u_{i}^{(2)}=\frac{F_{j}}{8 \pi \mu_{2}} \frac{2 \theta}{1+\theta}\left[\delta_{j \alpha}\left(\frac{\delta_{i \alpha}}{r}+\frac{r_{i}^{r} \alpha}{r^{3}}\right)+h \frac{\partial}{\partial x_{j}}\left\{\frac{-h r_{i}}{r^{3}}+\frac{\delta_{i 3}}{r}+\frac{r_{i} r_{3}}{r^{3}}\right\}\right] \text {, }
$$

(8b) $p^{(2)}=\frac{F}{4 \pi} \frac{2 \theta}{1+\theta}\left[\frac{\delta j \alpha^{p} \alpha}{r^{3}}+h \frac{\partial}{\partial x_{j}}\left(\frac{r}{r^{3}}\right)\right]$.

Here $\theta=\mu_{2} / \mu_{1}$ and $r$ and $R$ have been defined previously in (1a) and (1b). For the limiting cases of $\theta \rightarrow \infty$ and $\theta \rightarrow 0$ we obtain the results for the rigid no-sıip wall $(1 a),(1 b)$, and free surface $(2 a),(2 b)$, respectively. The case $\theta=1$ does not reproduce the infinite fluid solution because we have a distribution of normal stress over the surface specified by $x_{3}=0$, such that the normal velocity is zero (the imposed boundary condition).

It is perhaps easiest to understand the influence of the interface on 
the velocity and pressure fields to use the singularity diagrams of Blake [2]. The arrangement of the arrows for forces, couples, and doublets are reproduced in Figure 2. The analytic solutions given in $(7 \mathrm{a}),(\mathrm{Tb})$, and $(8 a),(8 b)$ are illustrated in the singularity diagrams of Figure 3 . The results are shown for Region I and Region II and in each case we consider singularity diagrams for tangential and normal forces. We will describe each case individually corresponding to the headings used in Figure 3.

\section{Singularity}

Stokeslet

\section{Representation}

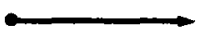

\section{order of magnitude}

$$
O\left(\frac{I}{p}\right)
$$

$$
0\left\{\frac{1}{r^{2}}\right\}
$$

Rotlet

(circular

Stresslet

(Radial

streamlines) streamlines)

\section{Symmetric}

Stokes-doublet

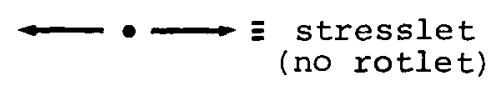

$$
0\left(\frac{1}{r^{2}}\right\}
$$

$$
\begin{array}{lcc}
\text { Stokes-quadrupole } & \text { (combinations of above) } & 0\left(\frac{1}{r}\right) \\
\text { Source-doublet } & \begin{array}{c}
\text { (special case of } \\
\text { Stokes-quadrupole) }
\end{array} & 0\left(\frac{1}{3}\right)
\end{array}
$$

FIGURE 2. Vector and tensor graphs of some singularities of the Stokes flow equations.

REGION I $\left(x_{3}<0\right.$, the lower half space $)$

(a) Tangential Force $\left(F_{\alpha}, \alpha=1\right.$ or 2$)$

In this case, the image system required to satisfy the boundary conditions consists of a stokeslet of strength $F_{\alpha}(1-\theta) /(1+\theta)$, a stokesdoublet of strength $-2 \theta h F_{\alpha} /(I+\theta)$ (in the figure a negative stokes- 
REGION 1

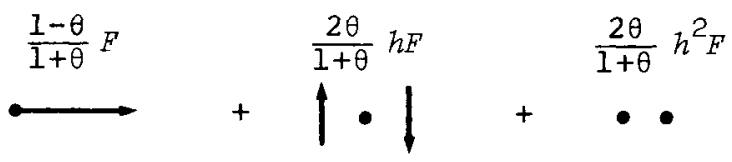

Image: Stokeslet Stokes- Sourcedoublet doublet

(a)

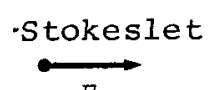

Far-field (i) $0 \leq \theta<\infty, \theta \neq 1 ; \frac{2}{1+\theta} F$ (stokeslet)

(ii) $\theta \rightarrow \infty$ (rigid wall); stresslet

Image: stokeslet stresslet source-doublet

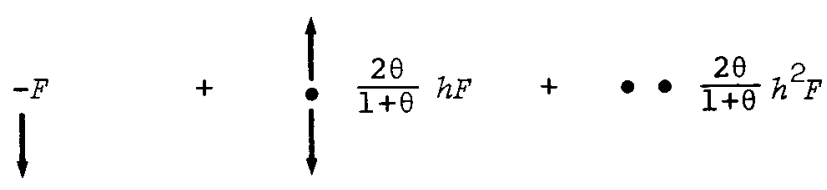

(b)

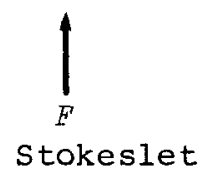

Far-field (i) $0 \leq \theta<\infty, \theta \neq 1 ; \frac{2 h F}{1+\theta}$, stresslet

(ii) $\theta \rightarrow \infty$ (rigid wall); stokes-quadrupole/ source doublet 
Region II

Far-field: stokeslet

(a)

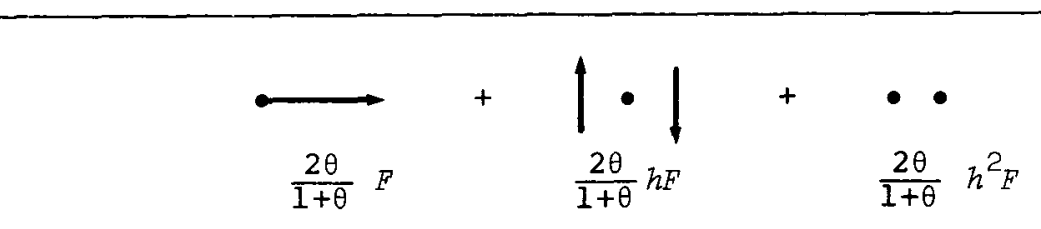

Image: stokeslet

stokes-

source-

doublet

doublet

Far-field: stresslet

(b)

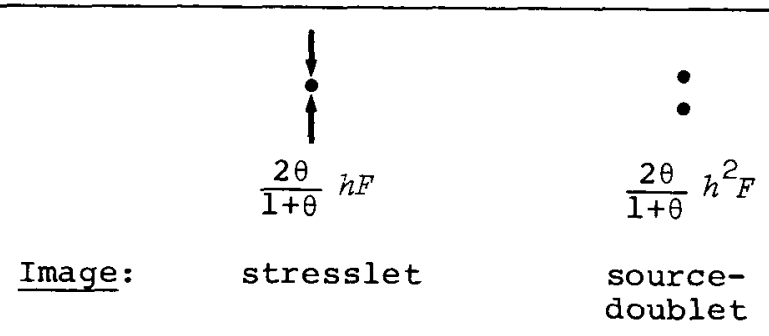

FIGURE 3. Singularity diagroms for Region I and Region II for tangential and normal force in Region I. The diagrams are explained in detail in the text. The far-fields in each case are shown on the diagram. 
doublet is illustrated) and a potential source doublet of strength $-2 \theta h^{2} F_{\alpha} /(1+\theta)$. The far-field for $0<\theta<\infty, \theta \neq 1$, consists of a stokeslet of strength $2 F_{\alpha} /(1+\theta)$ located at the origin. For the limiting case of a rigid, no-slip wall $(\theta \rightarrow \infty)$ the far-field consists of a stresslet, as discussed previously by Blake [2] and Blake and Chwang [4].

(b) Norma 1 Force $\left(F_{3}\right)$

The image system consists of an equal and opposite stokeslet $\left(-F_{3}\right)$, a symmetric stokes-doublet (stresslet) of strength $2 \theta h F_{3} /(1+\theta)$, and a source-doublet of strength $2 \theta h^{2} F_{3} /(1+\theta)$ for $0 \leq \theta<\infty, \theta \neq 1$. For the rigid wall case $(\theta \rightarrow \infty)$ the far-field becomes a combination of a stokes-quadrupole and a source-doublet.

REGION II $\left(x_{3}>0\right.$, the upper half space)

In this region there are no singularities within the half-space.

(a) Tangential Force

The singularities at the image point $(0,0,-h)$ consist of

(i) a stokeslet of strength $2 \theta F_{\alpha} /(1+\theta)$,

(ii) a stokes doublet of strength $-2 \theta h F_{\alpha} /(1+\theta)$, and

(iii) a source-doublet of strength $2 \theta h^{2} F_{\alpha} /(I+\theta)$.

Clearly, the far-field will behave like the stokeslet singuiarity except in the case where $\theta \rightarrow \infty$ in which case the upper fluid becomes a rigid solid.

(b) Normal Force $\left(F_{3}\right)$

In this case, the singularities required at the image point consist of

(i) a symetric stokes-doublet (stresslet) and

(ii) a potential source-doublet.

The far-field behaviour is that of a stresslet.

It should be emphasised that the far-field behaviour for a point force 
parallel to the wall is that of a stokeslet $(O(I / r)$ behaviour $)$ in both lower and upper fluids $(0 \leq \theta<\infty, \theta \neq 1)$, whereas in the case of a normal force it is a symmetric stokes-doublet $\left(O\left(1 / r^{2}\right)\right.$ behaviour $)$. In the case of a rigid wall $(\theta \rightarrow \infty)$, this changes to

(i) a stresslet far-field for the parallel stokeslet case, and

(ii) a stokes-quadrupole/source-doublet far-field for the normal force.

\section{First order approximation to interface shape}

To obtain the first-order approximations to the interface shape we have to solve the two-dimensional Poisson equation defined in (6). The logarithmic singularity prevents us obtaining a meaningful solution in the case of the normal force (another paradox in Stokes flow!), but for the case of a tangential force we obtain the expression for the interface shape

$$
\zeta=\frac{F^{h}}{2 \pi T} \frac{x_{\alpha}}{\rho^{2}}\left(1-\frac{h}{\left(\rho^{2}+h^{2}\right)^{\frac{3}{2}}}\right),
$$

where $\rho^{2}=x_{1}^{2}+x_{2}^{2}$.

For the case of a positive force, the fluid interface is depressed in the negative direction and increased on the positive side. The distortion of the interface is very important in helping us understand some of the possible surface changes that may occur when a particle approaches close to the interface. This is a surprising result because the surface shape is independent of $\theta$. However, we note that the total force acting on the free surface (the sum of the respective image forces) is equal to 1 .

In conclusion, this paper has presented the explicit expressions for the Green's functions due to a point force in one of the two half-space fluids in the case when inertial effects of the fluid are entirely negligible. The analytic expressions are discussed in terms of singularity diagrams and for the case of the tangential force a first approximation to the interface displacement is made. 


\section{References}

[1] K. Aderogba, "On stokeslets in a two-fluid space", J. Engrg. Math. 10 (1976), 143-151.

[2] J.R. Blake, "A note on the image system for a stokeslet in a noslip boundary", Proc. Cambridge Philos. Soc. 70 (1971), 303-310.

[3] John Blake, "On the movement of mucus in the lung", J. Biomech. 8 (1975), 179-190.

[4] J.R. Blake and A.T. Chwang, "Fundamental singularities of viscous flow. Part I: The image systems in the vicinity of a stationary no-slip boundary", J. Engrg. Math. 8 (1974), 23-29.

[5] John Happel, Howard Brenner, Low Reynolds number hydrodynamics with special applications to particulate media (Prentice-Hall, Englewood Cliffs, New Jersey, 1965).

[6] D.F. Katz, J.R. Blake and S.L. Paveri-Fontana, "On the movement of slender bodies near plane boundaries at low Reynolds number", $J$. Fluid Mech. 72 (1975), 529-540.

[7] L.D. Landau and E.M. Lifshitz, Fluid mechanies (Pergamon Press, oxfora, 1959).

[8] Sir James Lighthill, Mathematical biofluiddynamics (Society for Industrial and Applied Mathematics, Philadelphia, Pennsylvania, 1975).

[9] N.J. de Mestre, "Low-Reynolds-number fall of slender cylinders near boundaries", J. Fluid Mech. 58 (1973), 641-656.

[10] N.J. de Mestre, W.B. Russe|I, "Low-Reynolds-number translation of a slender cylinder near a plane wall", J. Engrg. Math. 9 (1975), $81-91$.

Engineering Analysis Unit,

CSIRO,

University of Lagos,

Division of Mathematics and Statistics,

Lagos, Canberra, ACT.

Nigeria; 